\title{
Reliability Assessment for the Solenoid Valve of a High-Speed Train Braking System under Small Sample Size
}

\author{
Jian-Wei Yang ${ }^{1 *}$, Jin-Hai Wang ${ }^{1,2}$, Qiang Huang ${ }^{3}$ and Ming Zhou ${ }^{1}$
}

\begin{abstract}
Reliability assessment of the braking system in a high-speed train under small sample size and zero-failure data is very important for safe operation. Traditional reliability assessment methods are only performed well under conditions of large sample size and complete failure data, which lead to large deviation under conditions of small sample size and zero-failure data. To improve this problem, a new Bayesian method is proposed. Based on the characteristics of the solenoid valve in the braking system of a high-speed train, the modified Weibull distribution is selected to describe the failure rate over the entire lifetime. Based on the assumption of a binomial distribution for the failure probability at censored time, a concave method is employed to obtain the relationships between accumulation failure probabilities. A numerical simulation is performed to compare the results of the proposed method with those obtained from maximum likelihood estimation, and to illustrate that the proposed Bayesian model exhibits a better accuracy for the expectation value when the sample size is less than 12. Finally, the robustness of the model is demonstrated by obtaining the reliability indicators for a numerical case involving the solenoid valve of the braking system, which shows that the change in the reliability and failure rate among the different hyperparameters is small. The method is provided to avoid misleading of subjective information and improve accuracy of reliability assessment under conditions of small sample size and zero-failure data.
\end{abstract}

Keywords: Zero-failure data, Modified Weibull distribution, Small sample size, Bayesian method

\section{Introduction}

The statistical model employed and the available sample size have a significant effect on the estimation of parameters in reliability assessment. A good statistical model provides a better fit to the actual lifetime data, and a good parameter estimation method can reduce the estimation error. For some time now, the normal distribution, lognormal distribution, exponential distribution, and Weibull distribution have served as commonly employed statistical models in reliability assessment. However, these models can only describe a particular type of failure rate. Lai et al. [1] developed a new model, denoted as the modified Weibull distribution, which included

\footnotetext{
*Correspondence: yangjianwei@bucea.edu.cn

1 Beijing Key Laboratory of Performance Guarantee on Urban Rail Transit Vehicles, Beijing University of Civil Engineering Architecture, Beijing 100044, China

Full list of author information is available at the end of the article
}

three parameters to control the shape of the curve, and employed this distribution to describe failure rates over the entire lifetime. Liu et al. [2] presented a new Bayesian model based on the Weibull distribution using a concave method and the relationships of failure probabilities in time, and verified the stability of the model via a practical application. Singh et al. [3] derived Bayesian estimations of hybrid censored lognormal distribution compared with maximum likelihood estimations and further computed Fisher information matrix, equal-tail and highest posterior density, and so on. Soliman et al. [4] studied the point and interval estimations of the modified Weibull distribution under squared error loss and linear exponential loss using the Markov chain Monte Carlo (MCMC) technique as an advanced algorithm to solve the problem of complex and high-dimensional integration for progressively type-II censored samples, and compared the results obtained based on two real data 
sets with those obtained using maximum likelihood estimation (MLE). Yang et al. [5] proposed a Bayesian reliability model under conditions of small sample size would have been applied to an NC machine tool, and a different method from that of MCMC would have been employed for high-dimensional integration. Xia [6] proposed a gray bootstrap method that can generate simulated data to compute reliability without other prior information for small sample size zero-failure data. Lord et al. [7] proposed a Poisson-gamma model describing motor vehicle crashes using Bayesian inference, and compared the results of numerical simulation with those obtained from MLE to demonstrate that the Bayesian estimation (BE) performed better than MLE. Fabrizi et al. [8] proposed a generalized inverse Gaussian prior for log-normal linear regression models and discussed how to choose parameters of the models under small and medium sample sizes. Junttila et al. [9] applied a Bayesian principal component regression model for remotely sensed data and verified it against other methods under spatial effects, multicollinearity and small size using an efficient Markov chain Monte Carlo sampling scheme. Hao et al. [10] proposed an improve Bayesian approach for precision system reliability assessment through searching for feasible points, screening feasible points and then simplifying the likelihood function. Ming et al. [11] presented the Bayesian model based on the mixed beta distribution. Comparing with traditional Bayesian analysis, the model presented considers differences between similar products. Jin [12] applied a bootstrap method for generating data to obtain the prior distribution and hierarchy of a Bayesian model describing the degradation process of space bearings for the purpose of calculating lifetime. A reliability evaluation method for segmented distribution using Bayesian method and an improved information criterion is proposed by Li et al. [13]. The method are proven more efficient and accurate than traditional method. Jia et al. [14] verified Bayesian estimation of multiply Type-I censored Weibull distribution is satisfactory for reliability assessment according to Monte Carlo simulation results. Peng et al. $[15,16]$ developed a Bayesian model updating approach to integrate subjective information in adjacent periods and in specific periods of lifetime stages, and applied the approach to assess a newly developed gantrytype machining center. Yang et al. [17] presented a comprehensive reliability allocation method based on cubic transformed functions, which can be designed to emphasize the failure severity or the failure occurrence depending on requirements. Salvinder et al. [18] employed a Markov chain model to represent the bending and torsion loads and estimated Weibull shape parameter to provide an accurate, efficient, fast and cost effective reliability analysis. Hamada et al. [19] and Cai et al. [20] have written relevant monographs regarding the use of Bayesian methods in reliability assessment that treat numerous topics such as advanced computation algorithms, model selection, degradation processes, and reliability growth analysis, and discuss several cases. Yao et al. [21] proposed a reliability assessment method based on T-S fault tree method and Bayesian network method, and compared with T-S fault tree method and Bayesian network method to prove feasible method.

Considering the hazardous nature of high-speed train operation, the assessment of vehicle reliability is essential to guarantee its safe operation. It is therefore necessary to assess the reliability of key components in high-speed trains, which represent issues that have been widely and thoroughly studied. Meng et al. [22] researched a reliability evaluation method using Markov model to describe the trend of the system reliability, and assessed the reliability of high-speed train traction transmission system. However, the system reliability needs research component reliability, which is statistical inference under small sample problem. Wang et al. [23] employed the least squares method to analyze the reliability of the key components of trains based on the Weibull distribution. The results of the study indicated that the sample size had a significant effect on the estimation precision. Wu et al. [24] assessed the reliability of relay valves based on the characteristic that the failure rate gradually increases according to an assumed Weibull distribution. Wang et al. [25] performed a durable test to evaluate the reliability of a brake unit for urban rail vehicles. The methods discussed above for the reliability assessment of high-speed trains can achieve satisfactory results under large sample size. Tian [26] employed a virtual expansion method to expand data sample size from $n=2-3$ to $n=12$, and then employed the method to analyze the reliability of the center sill and body bolster of a C70 gondola car based on a Bayesian method. The authors assumed that the lifetimes of parts followed normal distributions, and the approach was demonstrated to improve the assessment precision. Zhu et al. [27] extended the Bayesian method to the Weibull distribution, and verified the robustness of the model by assessing the reliability of bearings in a high-speed train. Subjective selection was employed to define the prior distribution and its hyperparameters, and these estimators were solved using an analytical method. Dong [28] assessed the reliability of braking systems in urban railway vehicles using a goal-oriented (GO) methodology and Bayesian theory based on the assumption of an exponential distribution. The method was demonstrated to be suitable for analyzing conditions where a component follows the rule that 


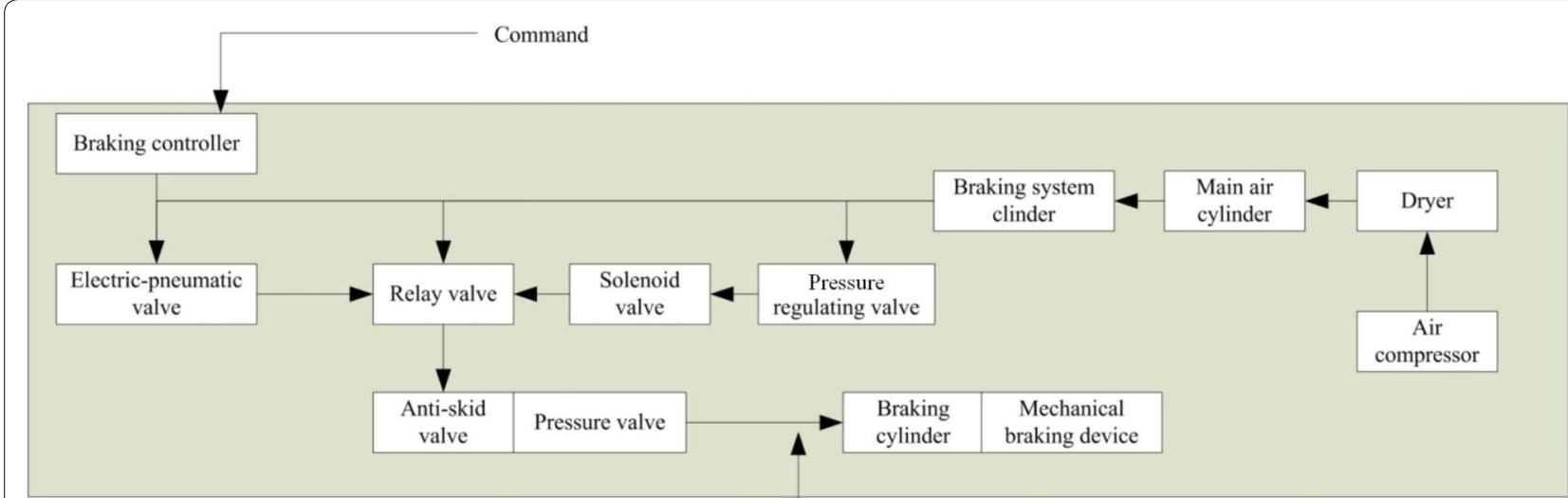

Oil

Figure 1 Structure of the braking system in high-speed trains

the failure rate is constant. Akama [29] calculated the crack propagation rate of cracks in a Shinkansen vehicle axle using Bayesian analysis by combining crack propagation rates derived from small specimens with crack propagation rates derived from full-scale models. The results showed that the method can narrow the variance of the fatigue life distribution, and provide more confident failure probability values.

Generally, reliability assessments are based on large sample size to attain statistical inferences (usually $n>30$ ). As such, reliability experiments consume considerable time and money. Owing to the long lifetimes, high cost, and complex structures of components in high-speed trains, it is necessary to develop reliability assessment theory and methods suitable under small sample size. The present study proposes a new approach that combines a Bayesian method with a subjective prior distribution for zero-failure data under small sample size, and experiments confirm that this approach can be safely applied to the reliability assessment of solenoid valves in the braking systems of highspeed trains. In Section 2, the failure model and the characteristics of zero-failure data for the solenoid valve are discussed. In Section 3, a Bayesian reliability model based on the binomial distribution at censored time is developed in detail. In Section 4, a modified Weibull distribution is introduced for the solenoid valve using least squares estimation based on failure probabilities at censored time. In Section 5, a numerical simulation is performed to compare the results of the proposed method with those of MLE. In Section 6, an actual numerical case is analyzed for the solenoid valve of the braking system in high-speed trains. Section 7 provides concluding remarks.

\section{Characteristics of the Solenoid Valve in the Braking System of High-Speed Trains}

The solenoid valve is an indispensable component in braking systems affecting the safety of railway vehicles. If the pressure of the braking system is sufficiently low, the solenoid valve will execute vehicle braking after arrival of the braking signal. Figure 1 illustrates the structure of the braking system in a high-speed train. The solenoid valve is of vital significance because of its intermediate position between the relay valve and the pressure regulating valve for ensuring the proper performance of emergency braking.

According to statistical data, the failure probability of the braking system in CRH1 EMU high-speed trains sharply increases when service distance is greater than $1.7 \times 10^{6} \mathrm{~km}$. Numerous conditions are responsible for the failure modes of the solenoid valve, such as conditions where the solenoid valve cannot be completely sealed due to internal valve fouling, power loss, or mechanical wear [30, 31].

As shown in Figure 2, the solenoid valve controls the action of a moveable iron core within a solenoid coil for

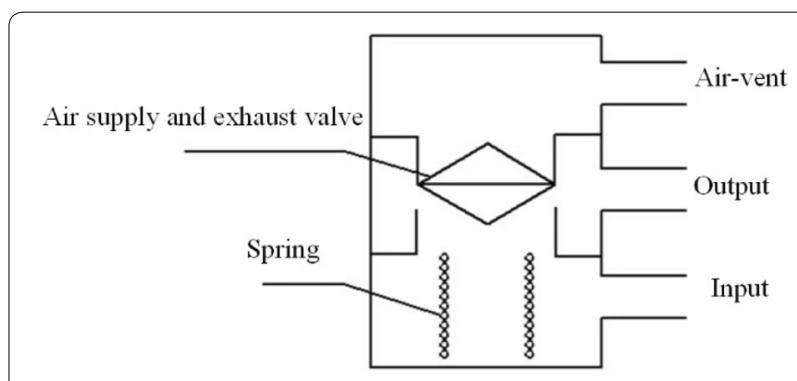

Figure 2 Structure of the solenoid valve 
opening or closing the exhaust valve through powering the solenoid coil on or off. It is obvious that the solenoid valve is a switch type component that is readily susceptible to wear-type failure. The accurate assessment of the reliability of the solenoid valve is essential.

\subsection{Reliability Assessment Model}

The modified Weibull distribution is a convenient model for describing particular failure rate function curves employed for components, and consists of three parameters that provide greater flexibility than the standard Weibull distribution [1]. The cumulative density function $F(t)$, reliability function $R(t)$, probability density function $f(t)$, and failure rate function $h(t)$ are given respectively as follows:

$$
\begin{aligned}
& F(t)=1-\exp \left[-\alpha t^{\beta} \exp (\lambda t)\right], \\
& R(t)=\exp \left[-\alpha t^{\beta} \exp (\lambda t)\right], \\
& f(t)=\alpha(\beta+\lambda t) t^{\beta-1} \exp (\lambda t) \exp \left[-\alpha t^{\beta} \exp (\lambda t)\right], \\
& h(t)=\alpha(\beta+\lambda t) t^{\beta-1} \exp (\lambda t),
\end{aligned}
$$

where $\alpha, \beta$, and $\lambda$ are the parameters of the modified Weibull distribution, where $\alpha>0, \beta \geq 0$, and $\lambda>0$.

The shape of $h(t)$ for different parameters is illustrated in Figure 3. The shape of $h(t)$ is defined by the parameter $\beta$, where, for $\beta \geq 1, h(t)$ increases with increasing $\mathrm{t}$ to describe a wearout period, and, for $0<\beta<1, h(t)$ provides a distinctive shape suitable for describing the lifetime of a component. The modified Weibull distribution reduces to the standard 2-parameter Weibull distribution when

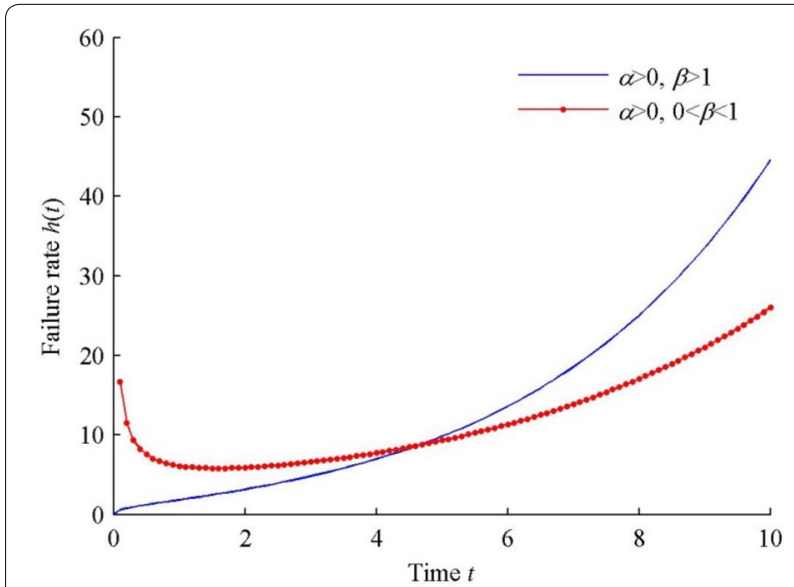

Figure 3 Shape of the failure rate curve for two different parameter settings for the modified Weibull distribution $\lambda=0$. In addition, the modified Weibull distribution reduces to the log-gamma distribution when $\beta=0$.

\subsection{Property of Failure Probability under Zero-Failure Data}

Liu et al. [2] researched the property of the failure probability pi and reliability assessment methods under the assumption of a Weibull distribution model for zero-failure data based on a concave method. In the present study, the concave method is applied for evaluating the relationships among $p_{i}$ for an assumed modified Weibull distribution. For lifetime experiments of the solenoid valve in high-speed train braking systems, the principle under zero-failure data can be described as follows.

(1) Assuming $s_{i}=m_{i}+m_{i+1}+\cdots+m_{k}$ is the number of the survival solenoid valve in total at $t_{i}$, where $m$ is the number of survival solenoid valve for censored time and it can be described as $\left(t_{i}, s_{i}\right)$.

(2) Assuming $p_{i}=P\left(T<t_{i}\right)=F\left(t_{i}\right), \quad i=1,2, \ldots, k$, it is obvious that $p_{i}$ is small, and $p_{1} \leq p_{2} \leq \cdots \leq p_{k}$.

(3) For $t=0, p_{0}=P(t=0)=0$.

Based on lifetime experiment data for a solenoid valve that does not fail, it is reasonable to consider $\beta \geq 1$.

Theory 1: for any $t_{i}, p_{i}$ satisfies the relation:

$$
0 \leq p_{i} \leq 1-\left(1-p_{k}\right)^{\frac{t_{i}}{t_{k}}}, \quad i=1,2, \ldots, k .
$$

Proof: $R(t)$ can be transformed to

$$
G(t)=-\ln R(t)=\alpha t^{\beta} \exp (\lambda t) .
$$

From the property discussed above, the followings are easily obtained:

$$
\begin{aligned}
& \frac{\partial G}{\partial t}=\alpha \beta t^{\beta-1} \exp (\lambda t)+\alpha \lambda t^{\beta} \exp (\lambda t), \\
& \frac{\partial^{2} G}{\partial t^{2}}=\alpha t^{\beta-2} \exp (\lambda t)\left[\lambda^{2} t^{2}+2 \beta \lambda t+\beta(\beta-1)\right], \\
& \because \alpha>0, \beta>1, \lambda>0, \\
& \therefore \frac{\partial G}{\partial t}>0, \frac{\partial^{2} G}{\partial t^{2}}>0 .
\end{aligned}
$$


From the above, we conclude that $G(t)$ is a monotonically increasing concave function. Therefore, from the property of a monotonically increasing concave function and $\ln R\left(t_{0}\right)=0$, we obtain

$$
\frac{-\ln R\left(t_{i}\right)}{t_{i}} \leq \frac{-\ln R\left(t_{k}\right)}{t_{k}} .
$$

Therefore,

$$
\left[R\left(t_{i}\right)\right]^{\frac{1}{t_{i}}} \geq\left[R\left(t_{k}\right)\right]^{\frac{1}{t_{k}}}, \quad i=1,2, \ldots, k .
$$

Substituting $R\left(t_{i}\right)=1-p_{i}$ into Eq. (5) yields

$$
0 \leq p_{i} \leq 1-\left(1-p_{k}\right)^{\frac{t_{i}}{t_{k}}}, \quad i=1,2, \ldots, k .
$$

Q.E.D.

\section{Assessment Method for Failure Probability Based on Bayesian Theory}

Bayesian theory can combine sample information with prior information to obtain a posterior estimator for more accurate result. To apply the MCMC method, the posterior distribution $h(\theta \mid x)$ must be described in the following form:

$$
h(\theta \mid x) \propto L(x \mid \theta) \times \pi(\theta),
$$

where $L(x \mid \theta)$ is a likelihood function, which contains all sample information for parameter $\theta$, and $\pi(\theta)$ is the prior distribution.

\subsection{Likelihood Function of Failure Probability for Zero-Failure Data}

It is assumed that $p_{i}$ follows a binomial distribution, and $L\left(r_{i} \mid p_{i}\right)$ can be written as

$$
L\left(r_{i} \mid p_{i}\right)=C_{s_{i}}^{r_{i}} p_{i}^{r_{i}}\left(1-p_{i}\right)^{s_{i}-r_{i}},
$$

where $C$ is a constant; $r_{i}$ is the number of failure sample. Under zero-failure data, $r_{i}=0$, and

$$
L\left(0 \mid p_{i}\right)=\left(1-p_{i}\right)^{s_{i}} .
$$

\subsection{Bayesian Inference}

\subsubsection{Bayesian Inference for Failure Probability $p_{k}$}

The critical step in Bayesian inference is to specify the prior distribution and its hyperparameters. For an informative distribution, the beta distribution is usually chosen to describe a parameter that is limited in $[0,1]$, such as reliability and failure probabilities, because the range of the beta distribution is from 0 to 1 and its shape is highly variable according to the value of its parameters. The beta distribution is illustrated in Figure 4 .

For zero-failure data, $p_{k}$ is generally very small. In other words, $p_{k}$ is more likely to be closer to 0 rather than 1 .

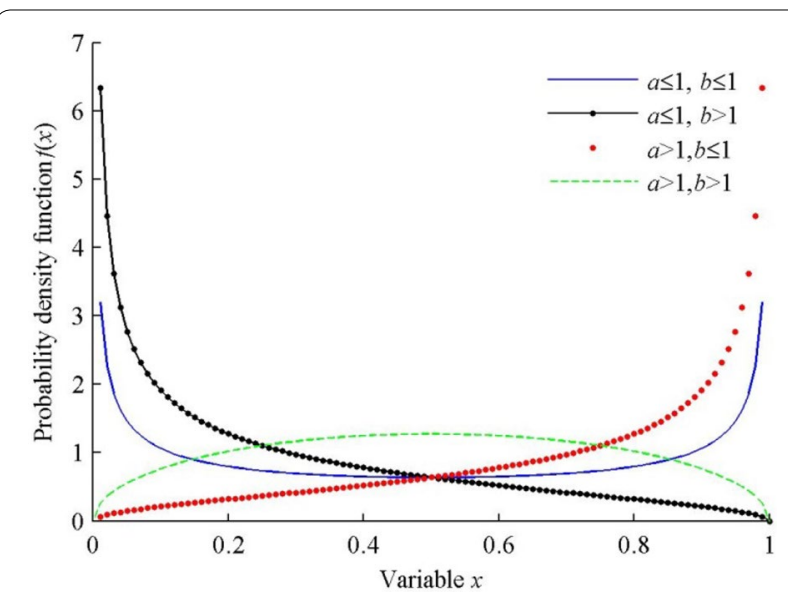

Figure 4 Shape of the beta distribution curve for different $a$ and $b$

Because of the characteristics of zero-failure data, the beta distribution curve is monotonically decreasing with $a \leq 1$ and $b>1$ according to actual conditions.

Under conditions lacking historical data or expert information, the non-discrimination principle is applied under the assumption of a uniform distribution as the prior distribution for parameters limited in a particular range. Although a uniform distribution is logical and convenient for defining a prior distribution of parameters, some problems, including boundedness and a dissatisfying invariance under variation for parameters, limit its application. To address these problems, the present study employs Jeffreys prior distribution and vague prior distribution rather than a uniform distribution. In this paper, it is assumed that $\pi\left(p_{k}\right)$ follows $\operatorname{Beta}(K \delta, K(1-\delta))$ as the prior distribution:

$$
\pi\left(p_{k} \mid K, \delta\right)=\frac{\Gamma(K)}{\Gamma(K \delta) \Gamma(K(1-\delta))} p_{k}^{K \delta-1}\left(1-p_{k}\right)^{K-K \delta-1},
$$

where $\delta$ is the mean value of $p_{k}$ and $K$ is the diffusion factor controlling the shape of the beta distribution. In this paper, it is assumed that $K \delta=1$, so that $K=1 / \delta$. Therefore, Eq. (9) can be given as

$$
\pi\left(p_{k} \mid \delta\right)=\frac{(1-\delta)}{\delta}\left(1-p_{k}\right)^{\frac{1-2 \delta}{\delta}} .
$$

For $\delta$, the hierarchical Bayesian method is applied to provide information using Beta distribution with parameters $\eta$ and $v$ due to its range from 0 to 1 . Thus, the hierarchical Bayesian model of $p_{k}$ is written as

$$
\pi\left(p_{k}, \delta \mid \eta, v\right) \propto \frac{(1-\delta)^{v}}{\delta^{2-\eta}}\left(1-p_{k}\right)^{\frac{1-2 \delta}{\delta}},
$$


where $\eta$ and $v$ are hyperparameters that can be calculated by numerous approaches such as the moment estimation approach and the percentiles approach. Substituting Eqs. (8) and (11) into Eq. (6), the posterior joint probability distribution can be obtained as

$$
h\left(p_{k}, \delta \mid \eta, v\right) \propto \frac{(1-\delta)^{v}}{\delta^{2-\eta}}\left(1-p_{k}\right)^{\frac{1-2 \delta}{\delta}+s_{k}}
$$

The Bayesian method mainly contains two problems. The first problem involves selecting the prior distribution and obtaining hyperparameters. The second problem is that it is nearly impossible to conduct the required integration for obtaining the normalizing constant of the posterior distribution for high-dimension calculations using numerical algorithms. To solve the second problem, the MCMC algorithm is applied to obtain the Bayesian estimator in this paper.

The MCMC algorithm provides a simple way to construct a Markov chain whose stationary distribution is the distribution of interest. From Eq. (12), the posterior distributions of the two parameters can be given by a posterior joint probability distribution as follows:

$$
\begin{aligned}
& h\left(p_{k} \mid \eta, v, \delta\right) \propto\left(1-p_{k}\right)^{\frac{1-2 \delta}{\delta}+s_{k}}, \\
& h\left(\delta \mid \eta, v, p_{k}\right) \propto \frac{(1-\delta)^{v}}{\delta^{2-\eta}}\left(1-p_{k}\right)^{\frac{1-2 \delta}{\delta}} .
\end{aligned}
$$

Based on Eqs. (13) and (14), the Bayesian estimators of $p_{k}$ and $\delta$ can be obtained using the MCMC algorithm.

\subsubsection{Bayesian Inference for Failure Probability $p_{i}$}

By Theory 1 , the value of $p_{i}(1 \leq i \leq k-1)$ can be limited to

$$
0 \leq p_{i} \leq 1-\left(1-p_{k}\right)^{\frac{t_{i}}{t_{k}}}, \quad i=1,2, \ldots, k .
$$

From a conservative perspective, the relationship between $p_{i}$ and $p_{k}$ can be defined as

$$
p_{i}=1-\left(1-p_{k}\right)^{\frac{t_{i}}{t_{k}}} .
$$

From Eqs. (13) and (15), the prior distribution of $p_{i}$ can be given as

$$
\pi\left(p_{\mathrm{i}} \mid \eta, \nu, \delta\right) \propto\left(1-p_{i}\right)^{\frac{(1-2 \delta) t_{k}}{\delta t_{i}}} .
$$

From Eqs. (6), (8), and (16), the posterior distribution of $p_{i}$ can be obtained as follows:

$$
h\left(p_{\mathrm{i}} \mid \eta, v, \delta\right) \propto\left(1-p_{i}\right)^{\left(\frac{1-2 \delta}{\delta}+s_{k}\right) \frac{t_{k}}{t_{i}} .}
$$

\section{Least Squares Estimation}

Based on the Bayesian estimators of $p_{i}$, the parameter values of the modified Weibull distribution can be calculated by the least squares method using. From Eq. (2), the modified Weibull distribution can be represented as a polynomial:

$$
y=\log (\alpha)+\beta \log (t)+\lambda t .
$$

From Eq. (18), the error of the least squares estimation can be described as the difference between the actual value and the target value with weights $\omega_{i}$ :

$$
\begin{array}{r}
Q(\alpha, \beta, \lambda)=\sum_{i=1}^{k} \omega_{i}\{\ln (\alpha)+\beta \ln (t) \\
\left.+\lambda t-\ln \left[-\ln \left(1-p_{i}\right)\right]\right\}^{2},
\end{array}
$$

where $\omega_{i}=n_{i} t_{i} / \sum_{i=1}^{k} n_{i} t_{i} \quad(i=1,2, \ldots, k)$ is the $i$ th weight value. The essence of least squares estimation is to minimize $Q(\alpha, \beta, \lambda)$ :

$$
Q(\hat{\alpha}, \hat{\beta}, \hat{\lambda})=\min _{\alpha>0, \beta>1, \lambda>1} Q(\alpha, \beta, \lambda)
$$

where $\hat{\alpha}, \hat{\beta}$ and $\hat{\lambda}$ are the least squares estimators of $\alpha$, $\beta$, and $\lambda$, respectively. These estimators are substituted respectively into Eqs. (2) and (4) to obtain $R(t)$ and $h(t)$.

\section{Numerical Simulation}

The numerical simulation was performed using an acceptance-rejection method to generate samples from a specific distribution whose parameters are fixed. Then, the estimators of all parameters obtained by the proposed Bayesian estimation (BE) were compared with those provided by MLE. In addition, the expectation value of the modified Weibull distribution, which is usually employed in reliability assessment to obtain the mean time between failures (MTBF), was calculated. Finally, the bias of each parameter was calculated.

The numerical simulation framework is given in detail by the following steps.

(1) Select a Weibull distribution $g(x \mid a, b)$ and a constant $M$ to satisfy $f(x \mid \alpha, \beta, \lambda) \leq M g(x \mid a, b)$.

(2) Generate a candidate sample $x^{\prime \prime}$ from $g(x \mid a, b)$.

(3) Generate a sample $u$ from $\operatorname{Unif}(0,1)$.

(4) Accept $x^{*}$ as a member of $f(x \mid \alpha, \beta, \lambda)$ if $u<f(x) / M g(x)$; otherwise, reject it.

(5) Repeat steps (1)-(4) until obtaining a sample size $n$. 
(6) Define $95,85,75 \%$ of the minimum among samples as the censoring time to obtain zero-failure data for size $n$.

(7) Calculate the estimators by the proposed $\mathrm{BE}$ and MLE.

(8) Repeat steps (1)-(7) 1000 times.

(9) Calculate the bias for each parameter group.

Based on the above steps, the simulations are divided into two groups with different parameters. Each group simulates 1000 sets of data with $n$ defined as 33, 24, 12 , and 6 , respectively. Based on each set of simulation data, the estimators are computed by BE and MLE. The simulations for each group of parameters are given as follows:

$$
\begin{aligned}
& \text { (1) } \alpha=1.5 \times 10^{-5}, \beta=1.5, \lambda=1.5 \times 10^{-5} \\
& \text { (2) } \alpha=1.5 \times 10^{-5}, \beta=1.5, \lambda=1 \times 10^{-5}
\end{aligned}
$$

Table 1 and Table 2 list the parameter bias results for the numerical simulations of the two groups. From a comparison of the results, we observe the following.

(1) For estimation of $\alpha, \mathrm{BE}$ always performs more poorly than MLE. For estimation of $\beta, \mathrm{BE}$ always performs better than MLE. For estimation of parameter $\lambda, B E$

Table 1 Simulation results for group 1

\begin{tabular}{lll}
\hline Parameters & Bayesian estimation & $\begin{array}{l}\text { Maximum } \\
\text { likelihood } \\
\text { estimation }\end{array}$ \\
\hline$n=33$ & $-6.21 \times 10^{-4}$ & $5.00 \times 10^{-6}$ \\
$a$ & $6.00 \times 10^{-2}$ & 5.77 \\
$\beta$ & $3.00 \times 10^{-7}$ & $-7.00 \times 10^{-2}$ \\
$\lambda$ & $8.94 \times 10^{2}$ & $5.76 \times 10^{2}$ \\
$E(t)$ & & \\
$n=24$ & $-3.97 \times 10^{-4}$ & $5.00 \times 10^{-6}$ \\
$a$ & $5.00 \times 10^{-2}$ & 5.46 \\
$\beta$ & $2.91 \times 10^{-7}$ & $-6.00 \times 10^{-2}$ \\
$\lambda$ & $7.53 \times 10^{2}$ & $4.15 \times 10^{2}$ \\
$E(t)$ & & \\
$n=12$ & $-2.28 \times 10^{-4}$ & $5.00 \times 10^{-6}$ \\
$a$ & $3.00 \times 10^{-2}$ & 4.95 \\
$\beta$ & $8.62 \times 10^{-7}$ & $-4.00 \times 10^{-2}$ \\
$\lambda$ & $3.66 \times 10^{2}$ & $-3.90 \times 10^{2}$ \\
$E(t)$ & & \\
$n=6$ & $-2.31 \times 10^{-4}$ & $5.00 \times 10^{-6}$ \\
$a$ & $4.50 \times 10^{-2}$ & 4.45 \\
$\beta$ & $1.97 \times 10^{-6}$ & $-2.00 \times 10^{-2}$ \\
$\lambda$ & $-3.70 \times 10^{2}$ & $-1.62 \times 10^{3}$ \\
$E(t)$ & & \\
\hline & &
\end{tabular}

Table 2 Simulation results for group 2

\begin{tabular}{lll}
\hline Parameters & Bayesian estimation & $\begin{array}{l}\text { Maximum } \\
\text { likelihood } \\
\text { estimation }\end{array}$ \\
\hline$n=33$ & $-6.08 \times 10^{-4}$ & $5.00 \times 10^{-6}$ \\
$a$ & $7.00 \times 10^{-2}$ & 5.85 \\
$\beta$ & $5.53 \times 10^{-6}$ & $-1.00 \times 10^{-1}$ \\
$\lambda$ & $8.97 \times 10^{2}$ & $6.00 \times 10^{2}$ \\
$E(t)$ & & \\
$n=24$ & $-4.27 \times 10^{-4}$ & $5.00 \times 10^{-6}$ \\
$a$ & $6.00 \times 10^{-2}$ & 5.47 \\
$\beta$ & $5.43 \times 10^{-6}$ & $-7.00 \times 10^{-2}$ \\
$\lambda$ & $7.27 \times 10^{2}$ & $5.82 \times 10^{2}$ \\
$E(t)$ & & $5.00 \times 10^{-6}$ \\
$n=12$ & $-1.55 \times 10^{-4}$ & 4.88 \\
$a$ & $3.00 \times 10^{-2}$ & $-4.00 \times 10^{-2}$ \\
$\beta$ & $5.69 \times 10^{-6}$ & $-5.12 \times 10^{2}$ \\
$\lambda$ & $2.92 \times 10^{2}$ & \\
$E(t)$ & & $5.09 \times 10^{-6}$ \\
$n=6$ & $-4.75 \times 10^{-4}$ & 4.42 \\
$a$ & $5.00 \times 10^{-2}$ & $2.00 \times 10^{-2}$ \\
$\beta$ & $6.46 \times 10^{-6}$ & $-1.62 \times 10^{3}$ \\
$\lambda$ & $-3.75 \times 10^{2}$ & \\
$E(t)$ & &
\end{tabular}

always performs better than MLE. For the expectation value, BE performs more poorly than MLE when $n$ is 33 and 24; However, with decreasing $n$, BE performs better than MLE, and always performs better for $n$ less than about 12 with regard to bias.

(2) With decreasing $n$, the estimation results for both methods improve for zero-failure data, although, for some cases, the bias values provided by BE and MLE do not follow this rule. This differs from the effects of complete data or other censoring data.

In conclusion, BE performs more poorly than MLE for estimating parameter $\alpha$, and performs better than MLE for the remaining parameters. In reliability assessment, the expectation is a vitally significant indicator for evaluating the durability and lifetime of a component, and affects the design of maintenance strategies. Moreover, the influence of the parameters on the shape of the reliability curve should be considered. Obviously, ensuring a high accuracy for $\beta$ and $\lambda$ is appropriate for controlling the curve shape. As such, the performance of the proposed Bayesian estimation method is more suitable than MLE for reliability assessment with zerofailure data under small sample size conditions. 


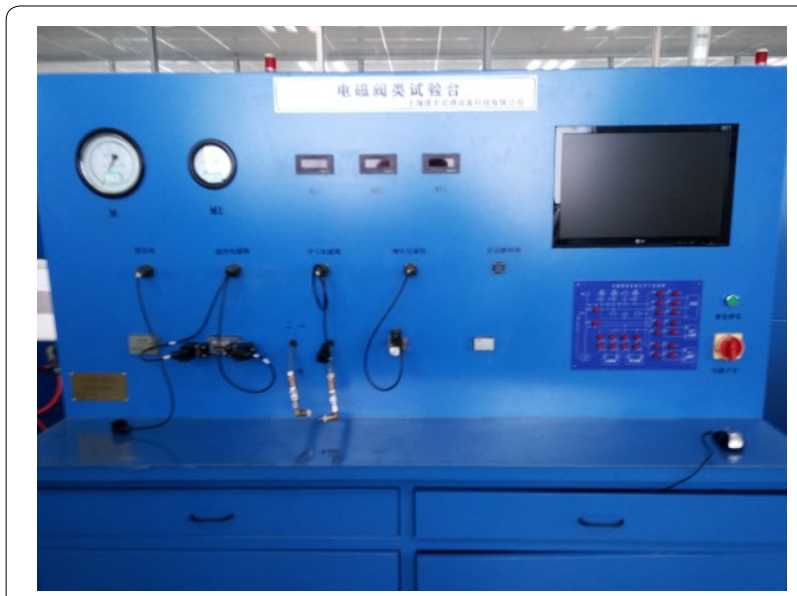

Figure $\mathbf{5}$ Test rig employed for lifetime experiments of solenoid valves

\section{Numerical Case}

\subsection{Lifetime Experiments for Solenoid Valves}

As discussed above, the solenoid valve of the braking system is a type of wearout product that plays a vital role in the system safety. Lifetime experiments were performed on a test rig to obtain lifetime data applicable to numerous types of solenoid valves employed in railway vehicles. An image of the test rig employed for the lifetime experiments is given in Figure 5.

In this case, a lifetime experiment performs on the test rig to observe the number and time of failure sample. The experimental framework of the test rig is illustrated in Figure 6. In this framework, a hydraulic source provides the motive force for controlling the solenoid valve to ensure the correct operation of the experiment under a range of frequencies. The test rig controls the
Table 3 Zero-failure data for solenoid valves

\begin{tabular}{llll}
\hline Number $\boldsymbol{i}$ & Data $\boldsymbol{t}_{\boldsymbol{i}}(\mathrm{h})$ & Quit number $\boldsymbol{m}_{\boldsymbol{i}}$ & $\begin{array}{l}\text { Survival } \\
\text { number } \boldsymbol{s}_{\boldsymbol{i}}\end{array}$ \\
\hline 1 & 800 & 2 & 18 \\
2 & 1000 & 2 & 16 \\
3 & 1200 & 2 & 14 \\
4 & 1500 & 2 & 12 \\
5 & 1750 & 3 & 9 \\
6 & 2000 & 3 & 6 \\
7 & 2150 & 3 & 3 \\
8 & 2300 & 3 & 0 \\
\hline
\end{tabular}

motion of the valve, and sends a signal reflecting the failure time to computer.

\subsection{Reliability Analysis for the Solenoid Valve}

A series of solenoid valve data with $n=\mathrm{z} 18$ was collected during reliability experiments. To simplify the calculation, the lifetime data was converted from counting number to hour, and the results are listed in Table 3.

Moment estimation was employed to obtain hyperparameters $\eta$ and $\nu$. We conservatively assigned $\delta=0.5$ based on the censored data obtained. Moreover, the robustness was evaluated because the variance of the assignment is an assumed value due to a lack of information. Therefore, three variance values were selected from the interval $[0.1,0.125]$, i.e., $0.1,0.1125$, and 0.125 . The failure probability results and the parameters of the modified Weibull distribution based on least squares estimation are listed in Table 4 and Table 5 , respectively. In addition, the effects of the different variance values on reliability and failure rate are illustrated in Figure 7.

The data in Table 4 shows that the estimators of each failure probability change very little when the variance is

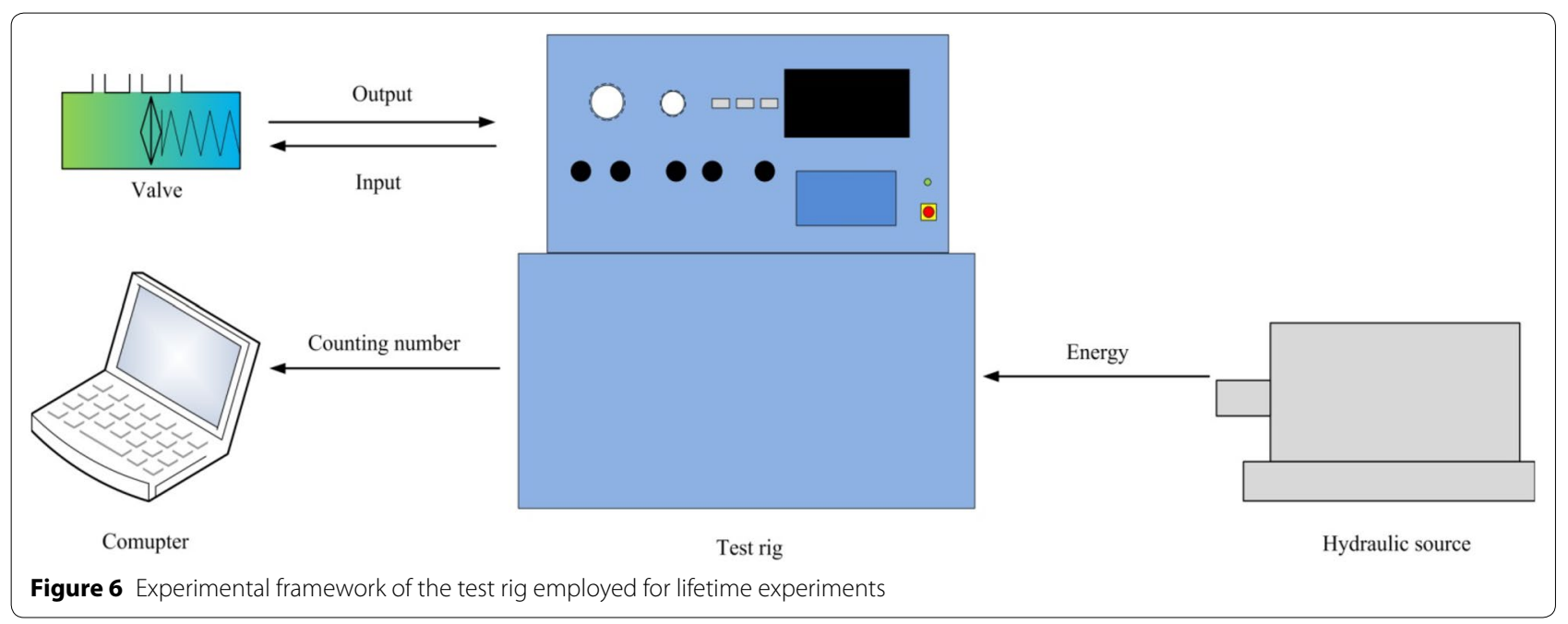


Table 4 Estimation of the failure probability in censored time under three different variance values (\%)

\begin{tabular}{llll}
\hline Failure probability & \multicolumn{3}{l}{ Variance value } \\
\cline { 2 - 4 } & $\mathbf{0 . 1}$ & $\mathbf{0 . 1 1 2 5}$ & $\mathbf{0 . 1 2 5}$ \\
\hline$p_{1}$ & 1.20 & 1.09 & 0.91 \\
$p_{2}$ & 1.50 & 1.36 & 1.13 \\
$p_{3}$ & 1.79 & 1.62 & 1.36 \\
$p_{4}$ & 2.22 & 2.01 & 1.69 \\
$p_{5}$ & 2.58 & 2.34 & 1.96 \\
$p_{6}$ & 2.93 & 2.66 & 2.23 \\
$p_{7}$ & 3.15 & 2.85 & 2.39 \\
$p_{8}$ & 3.36 & 3.04 & 2.55 \\
\hline
\end{tabular}

Table 5 Least square estimators of the modified Weibull distribution parameters under different variance values

\begin{tabular}{llll}
\hline $\begin{array}{l}\text { Estimators } \\
\text { of parameters }\end{array}$ & \multicolumn{2}{l}{ Variance value } & \\
\cline { 2 - 4 } & $\mathbf{0 . 1}$ & $\mathbf{0 . 1 1 2 5}$ & $\mathbf{0 . 1 2 5}$ \\
\hline$a$ & $1.3007 \times 10^{-5}$ & $1.7383 \times 10^{-5}$ & $1.9020 \times 10^{-5}$ \\
$\beta$ & 1.054 & 1.018 & 1.031 \\
$\lambda$ & $1.946 \times 10^{-6}$ & $1.101 \times 10^{-5}$ & $1.277 \times 10^{-5}$ \\
\hline
\end{tabular}

selected from the range [0.1, 0.125], and the maximum difference is only $0.81 \%$. Moreover, with increasing variance, the results are observed to increasingly approximate estimators based on sample data. This indicates that prior information becomes increasingly ambiguous with increasing variance, and reduces the impact of prior information on the posterior estimation.

Table 5 shows that the least squares estimators of the modified Weibull distribution parameters change very little when the variance is selected from [0.1, 0.125]. Moreover, as shown in Figure 7, the reliability and failure rate exhibit little variability with respect to the variance. This verifies that the modified Weibull distribution model employed in the Bayesian method based on the proposed prior is sufficiently robust.

Base on the results in Table 5, the reliability and failure rate functions of the modified Weibull distribution with a variance of 0.125 can be written as follows:

$$
\begin{aligned}
R(t)= & \exp \left[-1.902 \times 10^{-5} t^{1.031} \exp \left(1.277 \times 10^{-5} t\right)\right], \\
h(t)= & 1.902 \times 10^{-5}\left(1.031+1.277 \times 10^{-5} t\right) \\
& t^{0.031} \exp \left(1.277 \times 10^{-5} t\right) .
\end{aligned}
$$

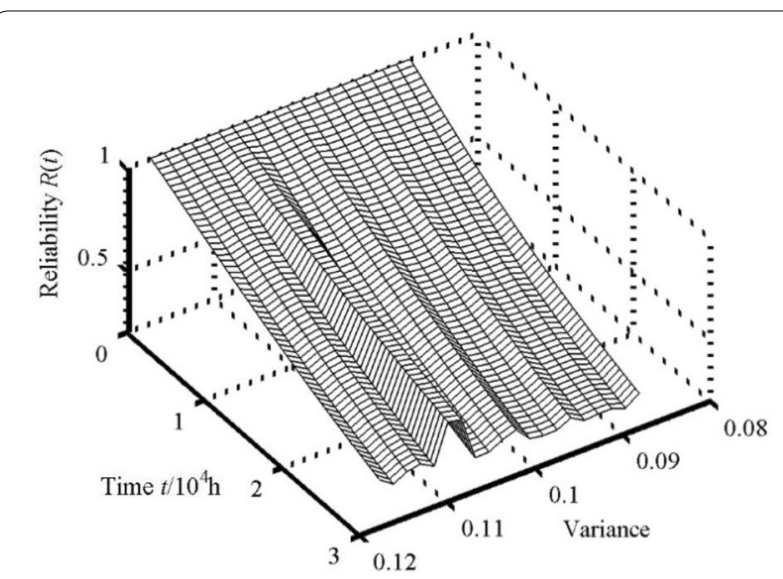

a Relation of the variance and time with reliability

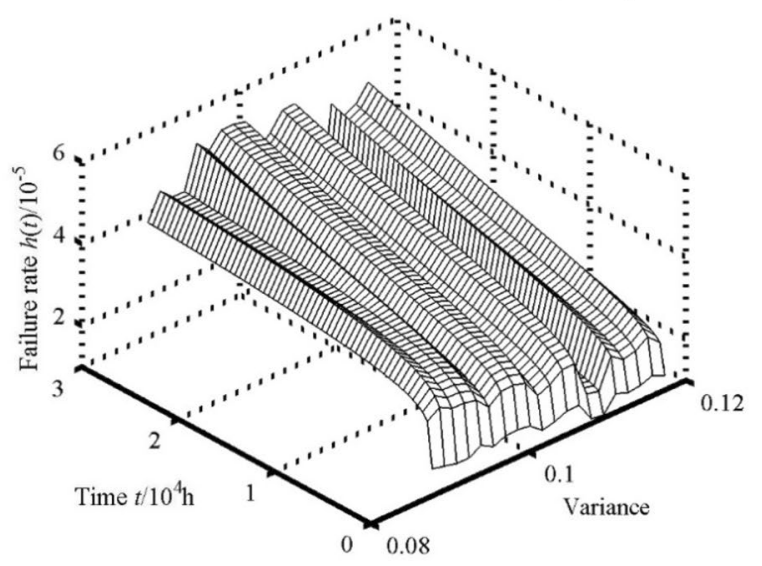

b Relation of the variance and time with failure rate

Figure 7 Relations of the variance and time with the reliability and failure rate

Then, Eq. (20) and Eq. (21) are plotted for $0 \mathrm{~h}$ to $20000 \mathrm{~h}$ in Figures 8(a) and (b), respectively.

The data presented in Figure 8(a) verifies the proper functioning of the algorithm, because the failure rate curve of the solenoid valve is monotonically increasing with increasing $t$ in accordance with the initial assumption. Meanwhile, the reliability of the solenoid valve remains greater than $50 \%$ after an operational period of 20000 h, as shown in Figure 8(b).

\section{Conclusions}

(1) The proposed Bayesian method is established based on engineering knowledge and mathematical relation. This method makes full use of objective information, and avoids misleading by vague subjective information. It reduces the difficulty of selecting parameters for reliability model. 

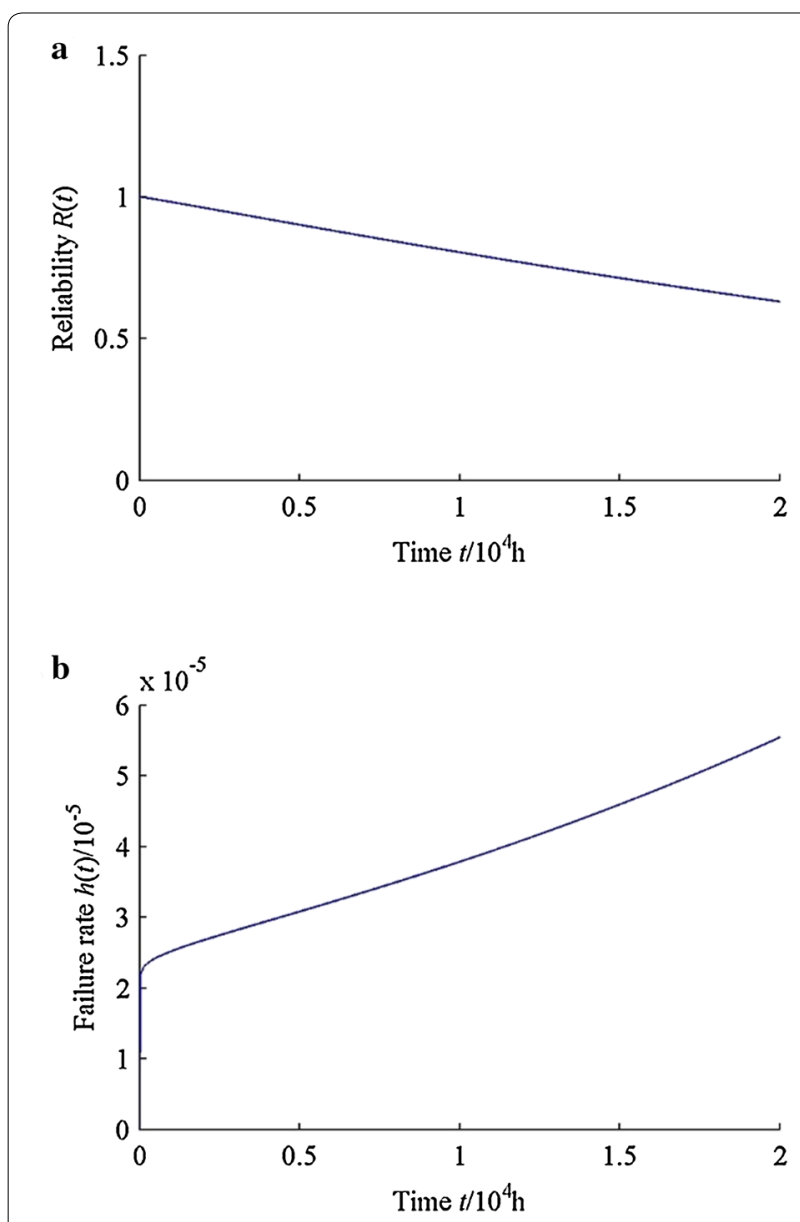

Figure 8 a reliability and $\mathbf{b}$ failure rate from Eqs. (20) and (21), respectively, for the solenoid valve in the braking system of a high-speed train

(2)A numerical simulation is investigated, and MLE method is chosen for comparison. The comparison indicates that the proposed Bayesian method can obtain more accurate results of the reliability assessment for the conditions of small sample size (less than 12) and zero-failure data.

(3) An application of the solenoid valve of the highspeed train braking system is introduced at the end of the paper. The results show the proposed Bayesian method can provide robust sufficiently estimations for different hyperparameters. The method is easier to apply than traditional method in the field of the reliability engineering of high-speed train.

\section{Authors' Contributions}

J-WY provided good experimental conditions and overall research objectives for the research work. J-HW put forward the research idea, wrote the manuscript, and carried out theoretical research and data analysis. QH assisted with the reliability testing. MZ improved the full text of english writing. All authors read and approved the final manuscript.

\section{Author details}

${ }^{1}$ Beijing Key Laboratory of Performance Guarantee on Urban Rail Transit Vehicles, Beijing University of Civil Engineering Architecture, Beijing 100044, China. 2 School of Mechanical, Electronic and Control Engineering, Beijing Jiaotong University, Beijing 100044, China. ${ }^{3}$ Locomotive and Car Research Institute, China Academy of Railway Science, Beijing 100044, China.

\section{Authors' Information}

Jian-Wei Yang, born in 1971, is currently a professor at Beijing University of Civil Engineering Architecture, China. He received his Ph.D. degree from China Academy of Railway Science, China, in 2006. His research interests include vehicle system dynamics, failure modeling analysis, system reliability and fault diagnosis. He is a senior member of CMES, Great Scholars Project and Beijing Recognized Talent Project. He has carried on many research work about reliability and risk assessment, e.g., The National Science Fund Project "The theory and analytical method study based on multi-state failure for braking system in high-speed train", China Postdoctoral Science Foundation Funded Project "Research on the reliability evaluation method of the vehicle system based on the small sample theory and GO method" and The National High Technology Research and Development Program of China "Research and verification of key equipment monitoring and early warning and emergency technology for rail transit operation safety". He has published about 130 papers in relative research field in total. Tel: +86-10-68322515; E-mail: yangjianwei@bucea.edu. $\mathrm{cn}$.

Jin-Hai Wang, born in 1990, is currently a Ph.D. candidate at School of Mechanical, Electronic and Control Engineering, Beijing Jiaotong University, China. He received his Master degree from Beijing University of Civil Engineering Architecture, China, in 2015. His research interests include reliability assessment, gearbox dynamics and mechanical fault diagnose. During the master period, he assisted his supervisor for studying reliability theory under small sample size, fatigue computation for structure of vehicle and optimization work. He also took part in Formula Student China 2015 and Honda energy competition for vehicle design. E-mail: wangjinhai@bjtu.edu.cn.

Qiang Huang, is a professor of China Academy of Railway Sciences, was born in 1946. He obtained his Master degree in Vehicle Engineering from China Academy of Railway Sciences, in 1981. His research interests are in vehicle system dynamics for heavy load railway vehicle, high-speed railway vehicle. He is also chief expert of China Academy of Railway Sciences and rewarded "Zhan Tianyou Railway Science and Technology award" in 2001. He carried on many research and development work for Chinese high-speed train, e.g., "Research for the bogie of 200 km/h EMU". E-mail: qhuang@rails.com.cn.

Ming Zhou, is an associate professor at Beijing University of Civil Engineering Architecture, was born in 1966. He obtained his Ph.D. degree in Mechanical Engineering from Dalian University of Technology, China, in 2006. His research interests are nonlinear dynamics and control.E-mail: zhouming@bucea.edu. $\mathrm{cn}$.

\section{Competing Interests}

The authors declare no competing financial interests.

\section{Funding}

Supported by National Natural Science Foundation of China (Grant No. 51175028), Great Scholars Training Project (Grant No. CIT\&TCD20150312) and Beijing Recognized Talent Project (Grant No. 2014018).

\section{Publisher's Note}

Springer Nature remains neutral with regard to jurisdictional claims in published maps and institutional affiliations.

Received: 18 January 2016 Accepted: 5 June 2018

Published online: 19 June 2018

\section{References}

[1] C D Lai, M Xia M, D N P Murthy. A modified Weibull distribution. IEEE Transactions on Reliability, 2003, 52(1): 33-37.

[2] HT Liu, Z H Zhang. Bayesian reliability analysis of Weibull zero failure data. Systems Engineering-Theory \& Practice, 2008, 28(11): 103-108. (in Chinese) 
[3] S Singh, Y M Tripathi. Bayesian estimation and prediction for a hybrid censored lognormal distribution. IEEE Transactions on Reliability, 2015, 65(2): 1-14

[4] A A Soliman, A H Abd-Ellah, N A Abou-Elheggag, et al. Modified Weibull model: A Bayes study using MCMC approach based on progressive censoring data. Reliability Engineering \& System Safety, 2012, 100(6): 48-57.

[5] Z Yang, Y Kan, C Fei, et al. Bayesian reliability modeling and assessment solution for NC machine tools under small-sample data. Chinese Journal of Mechanical Engineering, 2015, 28(6): 1229-1239.

[6] XXia. Reliability analysis of zero-failure data with poor information. Quality \& Reliability Engineering, 2012, 28(8): 981-990.

[7] D Lord, L F Miranda-Moreno. Effects of low sample mean values and small sample size on the estimation of the fixed dispersion parameter of Poisson-gamma models for modeling motor vehicle crashes: A Bayesian perspective. Safety Science, 2008, 46(5): 751-770.

[8] E Fabrizi, C Trivisano. Bayesian conditional mean estimation in LogNormal linear regression models with finite quadratic expected loss. Scandinavian Journal of Statistics Theory \& Applications, 2016, 43(4): 1064-1077.

[9] V Junttila, M Laine. Bayesian principal component regression model with spatial effects for forest inventory variables under small field sample size. Remote Sensing of Environment, 2017, 192: 45-57.

[10] Z P Hao, S K Zeng, J B Guo. Bayesian method for system reliability assessment of overlapping pass/fail data. Journal of Systems Engineering and Electronics, 2015, 26(1): 208-214.

[11] Z Ming, J Tao, X Chen, et al. Bayesian demonstration test method with mixed beta distribution. Chinese Journal of Mechanical Engineering, 2008, 21(3): 116-119.

[12] G Jin. Performance reliability modeling and estimation for space bearing under small sample circumstance. Journal of National University of Defense Technology, 2010, 32(1): 133-137.

[13] H Li, H Zuo, Y Su, et al. Study on segmented distribution for reliability evaluation. Chinese Journal of Aeronautics, 2017, 30(1): 310-329.

[14] X Jia, D Wang, P Jiang, et al. Inference on the reliability of Weibull distribution with multiply Type-I censored data. Reliability Engineering \& System Safety, 2016, 150: 171-181.

[15] W Peng, H Z Huang, Y Li, et al. Life cycle reliability assessment of new products-A Bayesian model updating approach. Reliability Engineering \& System Safety, 2013, 112(112): 109-119.

[16] W Peng, H Huang, Y Li, et al. Bayesian information fusion method for reliability assessment of milling head. Journal of Mechanical Engineering, 2014, 50(6): 185-191. (in Chinese)

[17] Z Yang, Y Zhu, H Ren, et al. Comprehensive reliability allocation method for CNC lathes based on cubic transformed functions of failure mode and effects analysis. Chinese Journal of Mechanical Engineering, 2015, 28(2): 315-324.

[18] S Salvinder, A Shahrum, N M N Abdullah, et al. Markov chain modelling of reliability analysis and prediction under mixed mode loading. Chinese Journal of Mechanical Engineering, 2015, 28(2): 1-8.

[19] M S Hamada, A G Wilson, C S Reese, et al. Bayesian reliability. New York: Springer, 2008

[20] H Cai, S F Zhang, J H Zhang. Bayes test analysis and assessment. Changsha: National University of Defence Technology Press, 2004. (in Chinese)

[21] C Yao, D Chen, B Wang, et al. Fuzzy reliability assessment method based on T-S fault tree and Bayesian network. Journal of Mechanical Engineering, 2014, 50(2): 193-201. (in Chinese)

[22] L Meng, Z Liu, L Diao, et al. Reliability evaluation of high-speed train traction transmission system based on Markov model. Journal of the China Railway Society, 2016, 38(8): 23-27.

[23] L Z Wang, Y G Xu, J D Zhang. Research on reliability analysis model for key components and parts of railway equipment and its application. Journal of the China Railway Society, 2008, 30(4): 93-97. (in Chinese)

[24] M L Wu, XY Wang, C Tian. Reliability of relay valve of brake system for rail vehicles. Journal of Southwest Jiaotong University, 2009, 44(3): 365-369. (in Chinese)

[25] X Y Wang, M L Wu. On the reliability of unit brake for urban rail vehicle. Urban Mass Transit, 2010, 13(11): 52-73. (in Chinese)

[26] Y Tian. Reliability analysis application for structure of beam of C70 railway vehicle under extreme small sample size. Beijing: Beijing Jiaotong University, 2008. (in Chinese)

[27] D X Zhu, H Z Liu. Reliability evaluation of high-speed train bearing with minimum sample. Journal of Central South University (Science and Technology), 2013, 44(3): 963-969. (in Chinese)

[28] J Z Dong. Study on urban rail vehicle brake system reliability modeling and simulation. Shanxi: Taiyuan University of Science and Technology, 2012. (in Chinese)

[29] M Akama. Bayesian analysis for the results of fatigue test using full-scale models to obtain the accurate failure probabilities of the Shinkansen vehicle axle. Reliability Engineering \& System Safety, 2002, 75(3): 321-332.

[30] W Chen. Study for frequent faults of brake system of CRH1A EMU. Conference Proceedings: China Railway Society Academic Committee for Trains, Passenger Vehicle, Qingdao: China Railway Society, 2012: 271-275. (in Chinese)

[31] $\mathrm{H}$ Zhang, $\mathrm{H}$ Jiang. The analysis of brake not releasing for $\mathrm{CRH} 5 \mathrm{EMU}$. Conference Proceedings: China Railway Society Academic Committee for Trains, Passenger Vehicle, Qingdao: China Railway Society, 2012: 269-270. (in Chinese)

\section{Submit your manuscript to a SpringerOpen ${ }^{\odot}$ journal and benefit from:}

- Convenient online submission

- Rigorous peer review

- Open access: articles freely available online

- High visibility within the field

- Retaining the copyright to your article

Submit your next manuscript at $\boldsymbol{\nabla}$ springeropen.com 